\title{
Dual-Mode Antenna for Health Monitoring System in Body-Centric Wireless Communications
}

\author{
Chia-Hsien Lin* and Koichi Ito \\ Center for Frontier Medical Engineering, Chiba University, \\ 1-33 Yayoi, Inage, Chiba 2638522, Japan
}

(Received March 3, 2014; accepted September 1, 2014)

Key words: Body-centric wireless communications (BCWCs), dual-mode antenna, on-body mode, off-body mode

Body-centric wireless communications (BCWCs) have become an active area of research owing to many applications, such as healthcare systems, identification systems, and entertainment. In this paper, a dual-mode antenna for body-centric wireless communications is studied. In on-body mode, the antenna is similar to a pair of metal electrodes operating at $10 \mathrm{MHz}$, and in off-body mode, with an L-shaped slit embedded, the antenna can cover the industrial, scientific, and medical (ISM) band (2.45 GHz). Since the proposed antenna is applied in BCWCs, the simulated received voltage and electric field distributions on the arm phantom, chest phantom, and high-resolution human model are discussed in this paper. Measured results, including reflection coefficient and radiation patterns in the ISM band $(2.45 \mathrm{GHz})$ are presented to validate the proposed antenna.

\section{Introduction}

In recent years, much attention has been paid to body-centric wireless communications (BCWCs) owing to their applications, such as healthcare systems, personal entertainment, smart home, and identification systems. Body-centric wireless communications are generally classified into three domains in accordance with the form of communication link, namely, on-body: communication between wearable devices; in-body: communication using implantable devices, and off-body: communication with external devices. The propagation properties of electromagnetic (EM) waves at different frequency bands will be described here. Figure 1 shows the simulated EM waves' properties on the human body at different operation frequencies. ${ }^{(1)}$ From the results, lowfrequency (tens of $\mathrm{MHz}$ ) signals propagate along the human body, while high-frequency (hundreds of $\mathrm{MHz}$ ) signals radiate outside the human body. Therefore, we will introduce a dual-mode antenna for medical application in body-centric wireless communications by using these two features.

${ }^{*}$ Corresponding author: e-mail: derek228015@gmail.com 


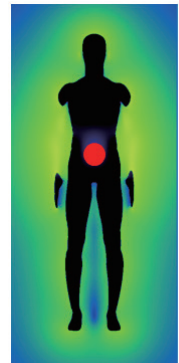

$3 \mathrm{MHz}$

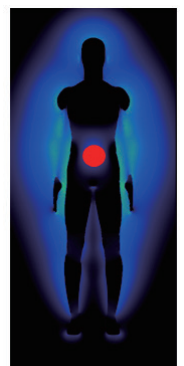

$30 \mathrm{MHz}$

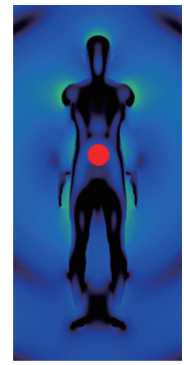

$300 \mathrm{MHz}$

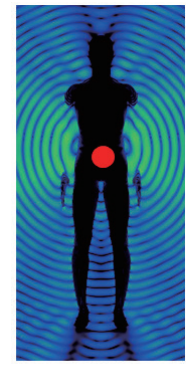

$3 \mathrm{GHz}$

Fig. 1. (Color online) Electric field distributions around the human body.

In practice, a single wireless device is usually expected to be applied in both onbody and off-body communications.(2) For example, we assume a medical care system, as presented in Fig. 2. The transmitters (sensors) and data controller (dual-mode antenna) are mounted on the human body. Medical information is transmitted to the data controller by the transmitter at low frequency $(10 \mathrm{MHz}) .^{(3-5)}$ After the information is received, the data controller sends it to an external device using the industrial, scientific, and medical (ISM) band $(2.45 \mathrm{GHz}) .{ }^{(6)}$ In this way, the health monitoring system can be achieved.

A dual-mode antenna for body-centric wireless communications is studied in this paper. This antenna can operate in two modes: on-body and off-body modes. In the onbody mode, the antenna is similar to a metal electrode operating at $10 \mathrm{MHz}$, and in the off-body mode, the antenna is similar to a slit-loaded antenna working in the ISM band (2.40-2.48 GHz). In our study, the characteristics of the antenna for on/off-body modes by using a muscle phantom at $10 \mathrm{MHz}$ and a two-thirds muscle phantom at $2.45 \mathrm{GHz}$ are discussed. Since the basic idea has been presented in our previous work, ${ }^{(7)}$ further study is provided in this paper.

In $\S 2$, the dual-mode antenna design and the human phantoms will be presented. In $\S \S 3$ and 4 , some important parameters, such as received voltage and electric field distribution for on-body mode and reflection coefficients and radiation patterns for offbody mode, are discussed. Finally, important conclusions and future works are outlined in $\S 5$.

\section{Antenna Design and Human Phantom}

\subsection{Dual-mode antenna design}

Figure 3 shows the proposed dual-mode antenna. It consists of a feeding pin, a signal electrode and an L-shaped slit embedded in the ground plane. Therefore, the proposed antenna is similar to a pair of metal electrodes. The areas of the ground plane and the electrode are $30 \times 36.5$ and $30 \times 10 \mathrm{~mm}^{2}$, respectively. The height of the signal electrode is $4 \mathrm{~mm}$, and the feeding pin is located at the center of the signal electrode. In order to integrate $2.45 \mathrm{GHz}$ ISM operation in the same antenna, an L-shaped slit of $2 \mathrm{~mm}$ width is embedded in the ground plane. 


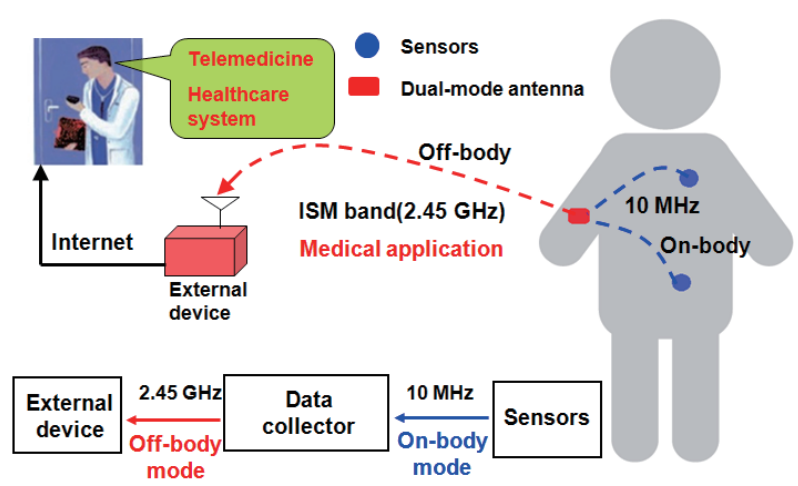

Fig. 2. (Color online) Image of proposed medical application.
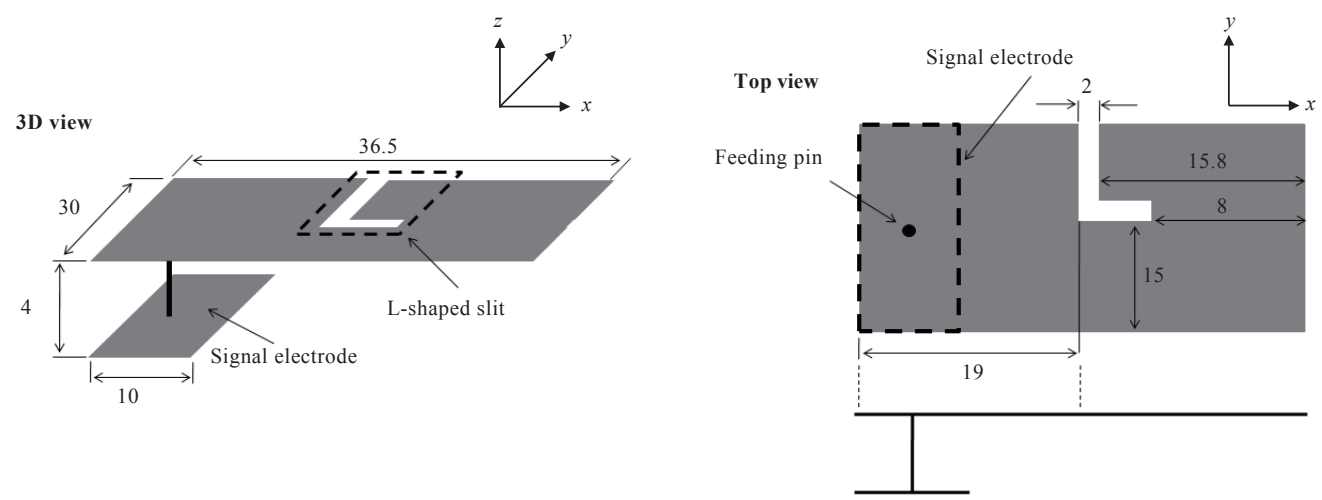

Fig. 3. Structure of proposed dual-mode antenna (unit: $\mathrm{mm}$ ).

\subsection{Human phantoms}

Since the proposed antenna is designed to support on-body and off-body communications, we should discuss the antenna characteristics in the proximity of two muscle-equivalent arm phantoms with the same size $\left(50 \times 50 \times 600 \mathrm{~mm}^{3}\right)$ at $10 \mathrm{MHz}$ (electrical constant $\varepsilon_{\mathrm{r}}=170.73$, conductivity $\sigma=0.62 \mathrm{~S} / \mathrm{m}$ ) and $2.45 \mathrm{GHz}$ (electrical constant $\varepsilon_{\mathrm{r}}=35.2$, conductivity $\sigma=1.16 \mathrm{~S} / \mathrm{m}$ ). The compositions of the two muscleequivalent phantoms at $10 \mathrm{MHz}$ and $2.45 \mathrm{GHz}$ are shown in Table 1. The fabricated phantoms are shown in Fig. 4.

\section{On-Body Mode}

In the on-body communication mode, since the communication is based on the quasistatic field, the received voltage is often adopted to evaluate the system. In fact, there are two typical scenarios in on-body mode: grounded system and ungrounded system. However, most of the medical applications use the ungrounded system. The scenario 
Table 1

Composition of two muscle-equivalent phantoms at $10 \mathrm{MHz}$ and $2.45 \mathrm{GHz}$.

\begin{tabular}{lll}
\hline & $\mathbf{1 0 ~} \mathbf{~ M H z}$ & $\mathbf{2 . 4 5} \mathbf{~ G H z}$ \\
\hline Material & Amount $[\mathrm{g}]$ & Amount $[\mathrm{g}]$ \\
\hline Water & 1291.2 & 3375 \\
Agar & 54 & 104.6 \\
Sodium chloride & 5.52 & 7 \\
Polyethylene powder & 0 & 1012.6 \\
TX-151 & 0 & 30.1 \\
Dehydroacetic acid sodium salt & 1.32 & 2 \\
\hline
\end{tabular}
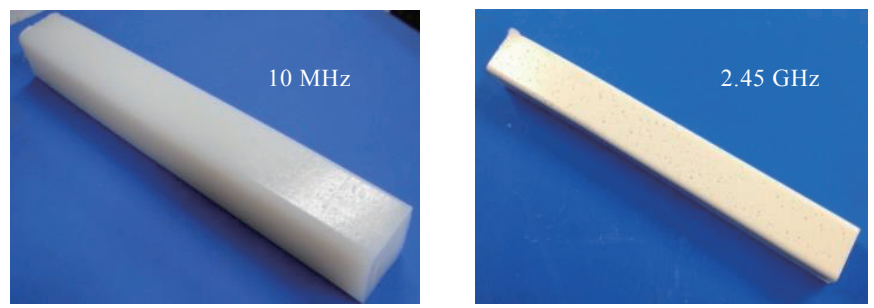

Fig. 4. (Color online) Fabricated arm phantoms for $10 \mathrm{MHz}$ and $2.45 \mathrm{GHz}$.

includes transmitting personal information by sensors that are attached to the human body. When the person's wearable sensors are mounted, his or her body signals, such as body temperature, blood pressure and heartbeat, can be detected and collected by the receiver.

In this section, the received voltage for the on-body mode is investigated. As shown in Fig. 5, the proposed transmitter and the receiver are both attached to the arm phantom. In this study, the assumption is that the transmitter is fed by an IC module, which can generate a sinusoidal signal of approximately $10 \mathrm{MHz}$. In addition, the finite-difference time-domain (FDTD) method is employed to calculate the received voltage and electric field distribution at $10 \mathrm{MHz}$ in this study. Table 2 shows the parameters for the calculations. The receiver was located $60 \mathrm{~mm}$ away from one end of the phantom and the distance between the transmitter and receiver was changed $(100,150,200,250,300$, 350,400 , and $450 \mathrm{~mm}$ ). Figure 6 shows the simulated received voltage and a polynomial fitting curve from MATLAB for the ungrounded system. From ref. 8, the capacitance coupling between the transmitter and the receiver is large and cannot be ignored for the ungrounded system. The capacitance coupling decreased and the impedance increased as the distance between the transmitter and the receiver was increased. Therefore, the increasing distance results in decreased receiver voltage between the transmitter and the receiver. Figure 7 shows the simulated electric field distribution for the ungrounded system. The result shows that the intensity of the electric field on the surface of the arm phantom decreased when the distance between the transmitter and the receiver increased. 


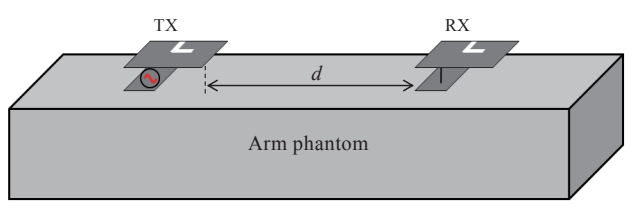

Fig. 5. (Color online) Illustration of ungrounded system.

Table 2

Parameters for simulation in FDTD.

\begin{tabular}{lc}
\hline Size of calculation space & $x \times y \times z=950 \times 500 \times 500 \mathrm{~mm}^{3}$ \\
Minimum cell size & $\Delta x=\Delta y=\Delta z=0.5 \mathrm{~mm}$ \\
Maximum cell size & $\Delta x=\Delta y=\Delta z=2 \mathrm{~mm}$ \\
Time step & $0.602 \mathrm{ps}$ \\
Iteration number & 200000 \\
Frequency range & $0-100 \mathrm{MHz}$ \\
Frequency & $10 \mathrm{MHz}$ \\
Pulse type & Gaussian derivative \\
Port type & Current source \\
\hline
\end{tabular}

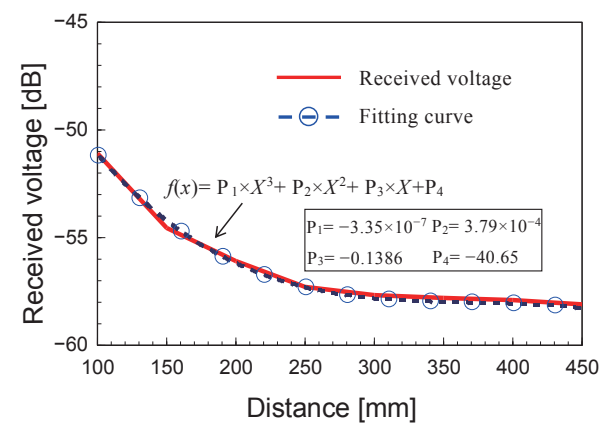

Fig. 6. (Color online) Simulated received voltage for an ungrounded system.

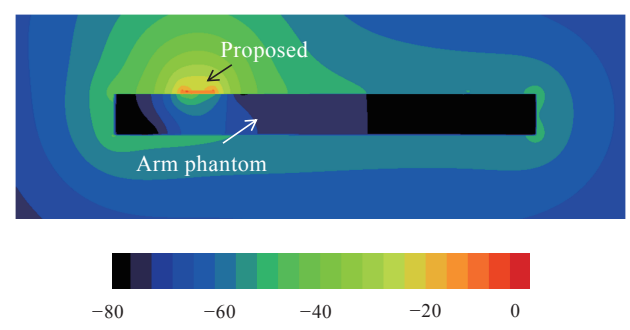

Fig. 7. (Color online) Electric field distribution at $10 \mathrm{MHz}$ on the arm phantom (unit: $\mathrm{dB}$ ). 
For this antenna, the difference in the received voltage caused by the structure of the proposed antenna will be discussed. The structure of the proposed antenna is changed according to the size and height of the electrode. Figure 8 shows the received voltage by increasing the width of the electrode from 5 to $15 \mathrm{~mm}$. From the result, it does not affect the received voltage because the impedance also changed. Figure 9 plots the received voltage when the height of the electrode is varied in the range of 2 to $6 \mathrm{~mm}$. From the result, the received voltage increased while the height of the electrode is increased. However, a low profile is an important issue in antenna design. Therefore, we adopted $4 \mathrm{~mm}$ for the height of the electrode in our study. From the results, the following conclusions are derived: (a) the received voltage decreases as the distance between TX (transmitter) and RX (receiver) increases, (b) the received voltage is not affected by the size of the electrode because the ground plane is larger than the electrode in our case, and (c) by increasing the height of the electrode, a higher received voltage could be obtained.

In this section, a whole human body model is also used to discuss the received voltage and the electric field distribution on the human body, as shown in Fig. 10. The calculations are based on a high-resolution human model from the National Institute of Information and Communications Technology (NICT) in Japan. In this study, the transmitter was fixed on the waist and the two receivers were located on the right and left sides of the chest and two more receivers on the right and left wrists. The height of the two human models is $1730 \mathrm{~mm}$, and there is a $30 \mathrm{~mm}$ gap between the two feet and the earth ground. From the simulation results shown in Fig. 11, owing to the shorter distance between the transmitter and the receiver, RX 3 obtained a higher received voltage than the other receivers. In addition, the received voltage of the simple human model is higher than that of the high-resolution model because the surface of the simple human model is flat and the signal electrode can be attached to the surface very well. The simulated electric field distribution of the high-resolution human body at $10 \mathrm{MHz}$ is shown in Fig. 12. From the result, the shapes of the contour lines of the electric field around the whole human body are smooth.
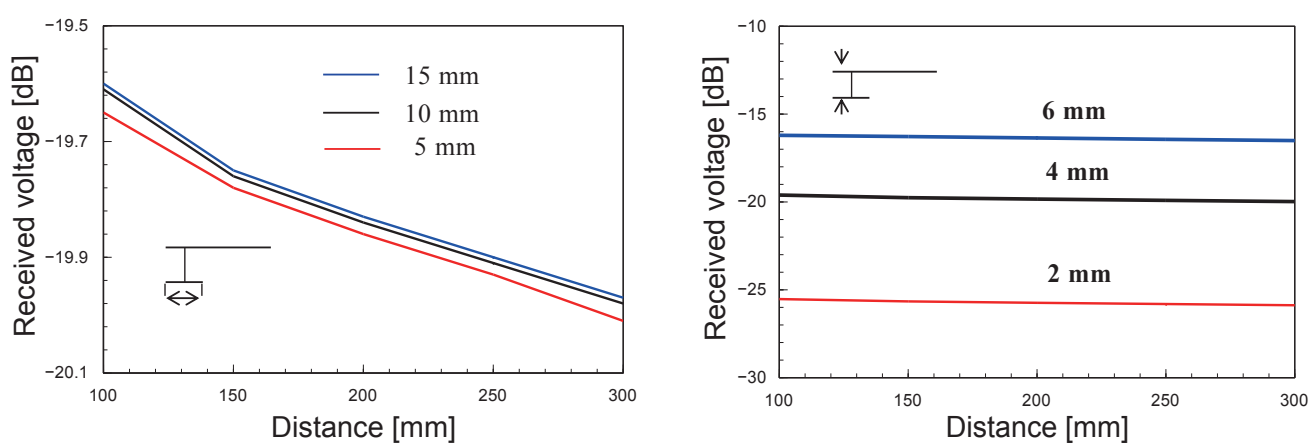

Fig. 8 (left). (Color online) Received voltage at $10 \mathrm{MHz}$ of increasing the width of the electrode. Fig. 9 (right). (Color online) Received voltage at $10 \mathrm{MHz}$ of varying the height of the electrode. 

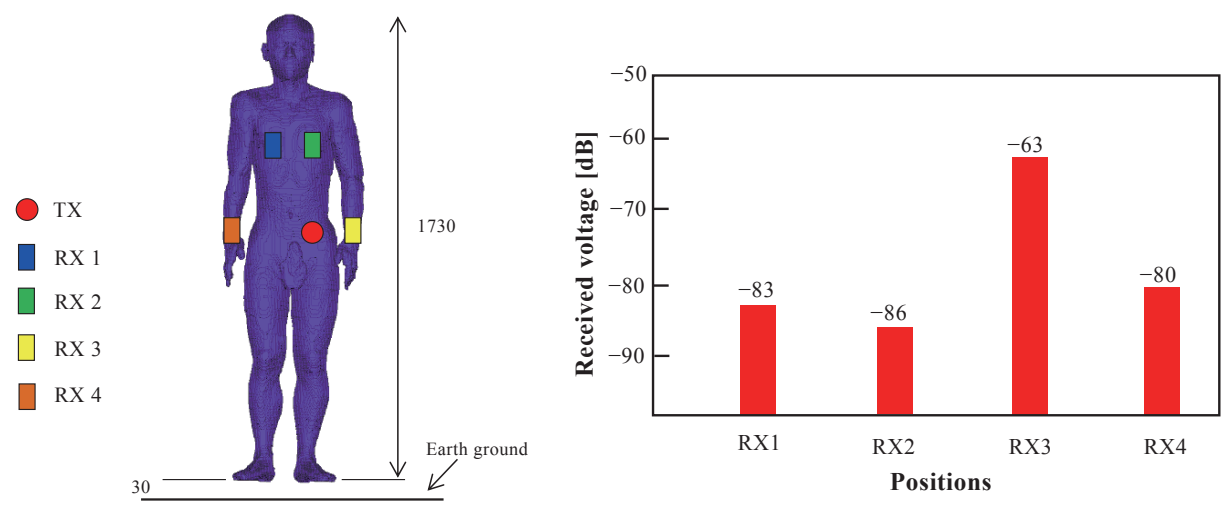

Fig. 10 (left). (Color online) Whole human model wearing the transmitter and receivers.

Fig. 11 (right). (Color online) Normalized simulated received voltage on the human model.

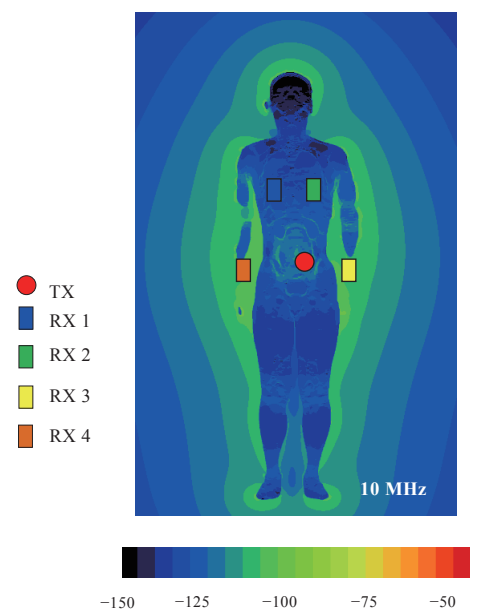

Fig. 12. (Color online) Electric field distribution of high-resolution human model (unit: dB).

\section{Off-Body Mode}

For off-body communication mode, we discuss reflection coefficients and radiation patterns in this section. Figure 13 shows the simulated and measured reflection coefficients in the $2.45 \mathrm{GHz}$ ISM band when the proposed antenna is on the arm phantom. The measured bandwidth is $320 \mathrm{MHz}(2.23-2.55 \mathrm{GHz})$ and the measured result is close to the simulated one. The slit embedded in the ground plane is the key element of the proposed antenna. To fully understand the slit of the proposed antenna, the slit width was analyzed. Figure 14 shows the simulated reflection coefficients with varying slit width. From the results, by tuning the width from 1 to $3 \mathrm{~mm}$, the resonant 

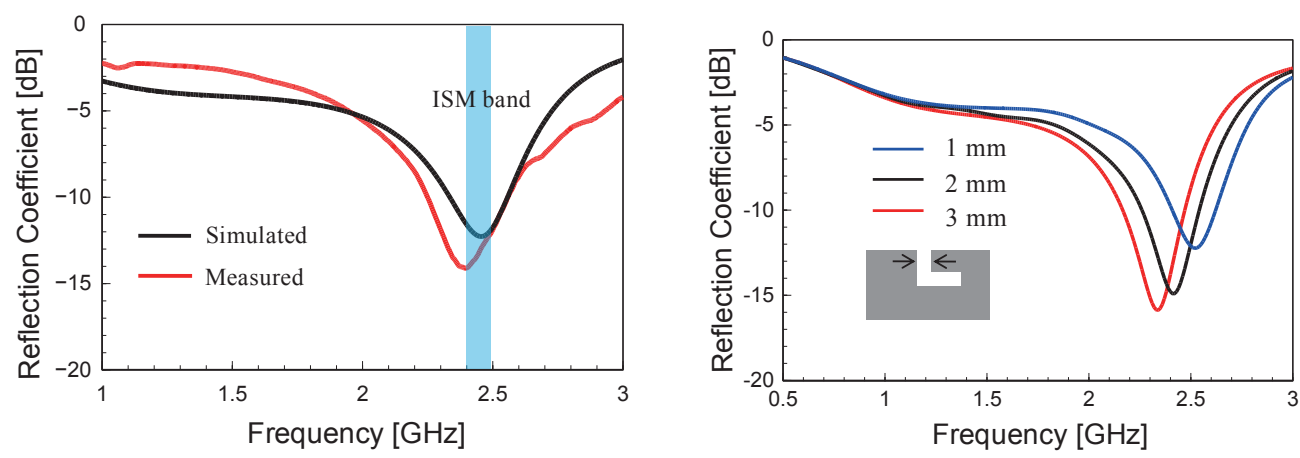

Fig. 13 (left). (Color online) Simulated and measured reflection coefficients.

Fig. 14 (right). (Color online) Simulated reflection coefficient with varying L-slit width.

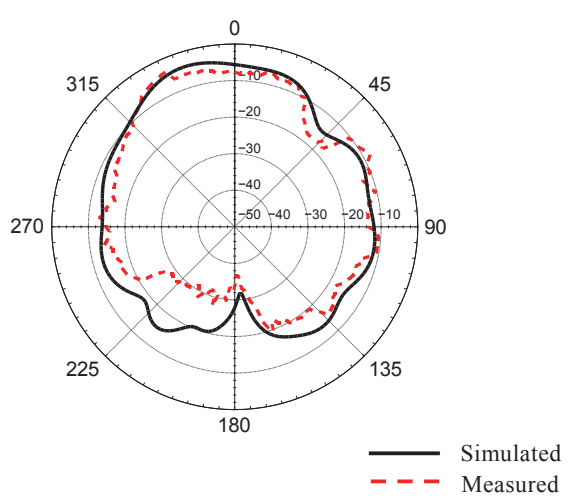

(a)

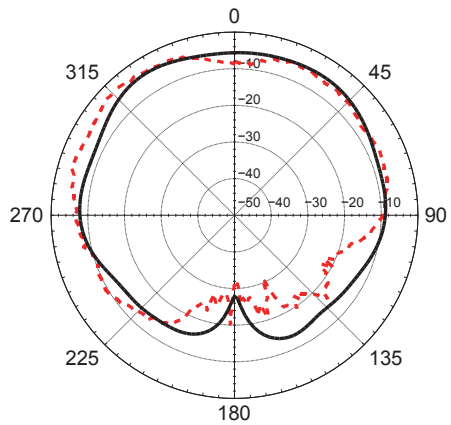

(b)

Fig. 15. (Color online) Simulated and measured radiation patterns (unit: dBi): (a) $x z$-plane and (b) $y z$-plane.

frequency decreases from 2.52 to $2.33 \mathrm{GHz}$. The proposed antenna works at $2.45 \mathrm{GHz}$ when the slit width is $2 \mathrm{~mm}$. The simulated and measured radiation patterns at 2.45 $\mathrm{GHz}$ in $x z$ - and $y z$-planes are shown in Fig. 15. From the results, the radiation patterns are relatively omnidirectional and there are no deep nulls in the half-sphere above the arm phantom. Therefore, the proposed antenna is a good candidate for off-body communications. In addition, the weaker radiation toward the phantom is due to the absorption by the phantom.

\section{Conclusions}

In this paper, a medical care system is assumed. The sensors and the data controller are mounted on the human body. Medical information is transmitted to the data controller by the transmitter at low frequency $(10 \mathrm{MHz})$, and the data controller sends 
it to an external device using ISM band at $2.45 \mathrm{GHz}$. To realize this idea, we proposed a dual-mode antenna in this paper. In this work, the received voltage and electric field distribution on the human body for on-body mode and the reflection coefficient and radiation patterns on the human phantom are discussed. The above studies confirmed that the proposed antenna can be applied to on-body and off-body communications. In the future, we will propose more practical and compact antennas for body-centric wireless communications in medical applications.

\section{References}

1 K. Ito, N. Haga, M. Takahashi and K. Saito: Proc. IEEE 100, 2012, p. 2356.

2 C. H. Lin, K. Ito, M. Takahashi and K. Saito: Proc. 2013 Int. Symp. Antenna Propag., 2013, p. 569.

3 K. Fujii, M. Takahashi and K. Ito: IEEE Trans. Antennas Propag. 55 (2007) 2080.

4 J. Wang, Y. Nishikawa and T. Shibata: IEEE Trans. Microwave Theory Tech. 57, 2009, p. 2464.

5 T. G. Zimmerman: IBM Syst. J. 35 (1996) 609.

6 C. H. Lin Lin, K. Saito, M. Takahashi and K. Ito: IEEE Trans. Antennas and Propag. 60 (2012) 4422.

7 C. H. Lin, Z. Li, K. Ito, M. Takahashi and K. Saito: Electron. Lett. 48 (2012) 1383. 